\title{
Cambios regionales de densidad vascular parafoveal en pacientes diabéticos sin retinopatía
}

\author{
Sheyla L. Escobar-Villado y Virgilio Lima-Gómez ${ }^{2 *}$ \\ ${ }^{1}$ Universidad Autónoma de Ciudad Juárez, Escuela de Medicina, Chihuahua; ${ }^{2}$ Hospital Juárez de México, Servicio de Oftalmología, Ciudad de \\ México. México
}

\section{Resumen}

Introducción: Los cambios regionales de densidad vascular parafoveal no han sido caracterizados en los pacientes con diabetes tipo 2 sin retinopatía. Objetivo: Comparar los cambios regionales de densidad vascular entre sujetos sin diabetes (grupo 1) y con diabetes sin retinopatía (grupo 2). Métodos: Estudio observacional, retrospectivo, transversal y comparativo. La densidad vascular parafoveal por cuadrantes se comparó entre grupos y entre cuadrantes en cada grupo; se correlacionó la densidad de cada cuadrante y la densidad interna, así como la contribución de cada cuadrante a la densidad interna (regresión lineal). Resultados: Se analizaron 34 ojos en el grupo 1 y 38 en el grupo 2; en este fue menor la densidad vascular interna (20.75 versus $21.85, p=0.021$ ) y la de los cuadrantes superior (21.05 versus $21.75, p<0.001$ ) y nasal (20.95 versus $21.50, p=0.023$. En el análisis de regresión, los cuadrantes superior y nasal tuvieron la mayor contribución al cambio en la densidad vascular interna $(\beta=0.361, \beta=0.294)$ en el grupo 2. Conclusiones: La densidad vascular interna parafoveal disminuye en los pacientes diabéticos sin retinopatía, a expensas de los cuadrantes superior y nasal, por lo que se requiere evaluación adicional para detectar afecciones microvasculares tempranas.

PALABRAS CLAVE: Densidad vascular parafoveal. Diabetes tipo 2. Retinopatía diabética. Angiografía por tomografía de coherencia óptica.

\section{Regional parafoveal vessel density changes in diabetic patients without retinopathy}

\section{Abstract}

Introduction: Regional parafoveal vessel density changes have not been characterized in patients with type 2 diabetes without retinopathy. Objective: To compare regional changes in vessel density between subjects without diabetes (group 1) and with diabetes without retinopathy (group 2). Methods: Observational, retrospective, cross-sectional, comparative study. Parafoveal vessel density by quadrants was compared between groups and between quadrants in each group; density of each quadrant and inner density were correlated, as well as the contribution of each quadrant to inner density (linear regression). Results: Thirty four eyes were analyzed in group 1 and 38 in group 2; in the latter, inner vessel density (20.75 vs. 21.85, $p=0.021)$ and that of superior quadrants (21.05 vs. $21.75, p<0.001)$ and nasal quadrants $(20.95 \mathrm{vs} .21 .50, p=0.023)$ were lower. In the regression analysis, superior and nasal quadrants had the largest contribution to the change in inner vessel density in group 2 ( $\beta=0.361$ and $\beta=0.294$, respectively). Conclusions: Parafoveal inner vessel density decreases in diabetic patients without retinopathy at the expense of superior and nasal quadrants, which indicates that further evaluation is required in order for early microvascular disease to be detected.

KEY WORDS: Parafoveal vessel density. Type 2 diabetes. Diabetic retinopathy. Optical coherence tomography angiography.

Correspondencia:

*Virgilio Lima-Gómez

E-mail: forscher7@gmail.com
Gac Med Mex. 2021;157:352-356

Disponible en PubMed

www.gacetamedicademexico.com

CC BY-NC-ND (http://creativecommons.org/licenses/by-nc-nd/4.0/). 


\section{Introducción}

La retinopatía diabética $(\mathrm{RD})$ es la causa principal de ceguera en edad laboral a nivel mundial. El riesgo de pérdida visual en personas con diabetes es $25 \%$ más alto que en las personas sin ella. ${ }^{1}$ La hiperglucemia desempeña un papel importante en la patogénesis del daño microvascular en la retina; las primeras respuestas de esos vasos sanguíneos a la hiperglucemia son la dilatación y los cambios en su flujo. ${ }^{2}$

Hasta hace poco, el diagnóstico del daño retiniano causado por la diabetes se limitaba a la oftalmoscopia, la fotografía de fondo de ojo y la angiografía con fluoresceína. ${ }^{3}$ La angiografía por tomografía de coherencia óptica (OCT-A) es una tecnología desarrollada recientemente que detecta el movimiento de los eritrocitos y permite delimitar la microvasculatura retiniana, con una resolución equiparable a la de un corte histológico, por lo cual es una alternativa no invasiva para investigar la retinopatía diabética. ${ }^{4,5}$

El equipo de angiografía por tomografía de coherencia óptica Cirrus $5000^{\mathrm{TM}}$ con Angioplex ${ }^{\mathrm{TM}}$ (Zeiss, Meditec, Dublín, CA) puede generar un mapa de $3 \times 3 \mathrm{~mm}$ y medir la densidad vascular en la región central de la mácula, el área de mejor visión. Este equipo determina automáticamente la densidad vascular del plexo capilar superficial en el área central (un círculo central de $1 \mathrm{~mm}$ ) y en un anillo de $1 \mathrm{~mm}$ hacia fuera de ella; también mide la densidad completa de los $3 \mathrm{~mm}$ centrales y puede dividir al anillo en cuadrantes: superior, temporal, inferior y nasal, para cada uno de los cuales registra la densidad vascular. ${ }^{6}$

Se ha identificado una reducción de la densidad vascular parafoveal en el plexo superficial y profundo en pacientes diabéticos sin evidencia clínica de retinopatía diabética, ${ }^{7}$ que se acentúa conforme aumenta la duración de la diabetes. ${ }^{8}$ Sin embargo, se desconoce cómo contribuyen a ella las reducciones regionales de densidad vascular y si alguna es más marcada, lo cual podría indicar una afección selectiva anticipada. Se diseñó un estudio para comparar los cambios regionales en la densidad vascular parafoveal entre sujetos sin diabetes y pacientes con diabetes sin retinopatía, a fin de determinar si hay sectores con mayor daño, en quienes pudiera hacerse una detección más temprana.

\section{Métodos}

Se realizó un estudio observacional, retrospectivo, transversal y comparativo. La población objetivo fueron sujetos sin diabetes y pacientes con diabetes tipo 2 , sin retinopatía diabética de la Ciudad de México y su área metropolitana. La población accesible fueron los pacientes tratados en el servicio de oftalmología de un hospital federal de referencia. El estudio se realizó del 3 de julio al 3 de agosto de 2020, se apegó a los principios de la Declaración de Helsinki y recibió la autorización de los comités de investigación y ética en investigación del hospital donde se desarrolló.

Se incluyeron sujetos sin diabetes y pacientes con diabetes tipo 2 sin retinopatía diabética, de uno u otro sexo, con edad de 30 a 70 años, con cualquier grado de agudeza visual corregida, que contaran con un mapa de angiografía por tomografía de coherencia óptica de adecuada calidad. Se excluyeron los pacientes con cualquier otro tipo de enfermedad de la retina o con opacidades de los medios oculares. Se eliminaron los pacientes con valores fuera de \pm 3 desviaciones estándar del grosor retiniano.

La muestra se dividió en dos grupos: 1, sin diabetes; 2, con diabetes sin retinopatía diabética. Se incluyó un solo ojo por sujeto, que se seleccionó de forma aleatoria en los pacientes que cumplieron con los criterios de selección.

Las imágenes de angiografía por tomografía de coherencia óptica se obtuvieron con el equipo Cirrus $5000 \mathrm{HD}$ con Angioplex. Las características de los estudios fueron cubo macular de $6 \mathrm{~mm}$ de alta resolución y angiograma del plexo capilar superficial de $3 \times 3 \mathrm{~mm}$, que se adquirieron mediante el algoritmo de segmentación automática del equipo; los controles de calidad de las imágenes incluyeron un centrado correcto en la fóvea, la ausencia de artefactos de parpadeo o movimiento y una intensidad de señal $>7$.

La variable en estudio fue la densidad vascular parafoveal del plexo superficial $\left(\mathrm{mm}^{-1}\right)$, que se midió por regiones (central, interna y completa), la densidad interna se midió por cuadrantes: superior, inferior, temporal y nasal. Las variables secundarias fueron el grosor retiniano (en $\mu \mathrm{m}$ ) y el volumen macular (en $\mathrm{mm}^{3}$ ). Todas las mediciones se calcularon automáticamente por el equipo.

Las variables numéricas se reportaron como mediana y rango intercuartílico. La densidad vascular parafoveal se comparó entre grupos mediante la prueba U de Mann-Whitney; la densidad vascular se comparó entre cuadrantes en cada grupo mediante la prueba Kruskal-Wallis. En cada grupo se identificó la correlación entre las densidades de cada cuadrante y la densidad interna mediante la prueba rho de Spearman. Se realizó un análisis multivariado mediante 
Tabla 1. Comparación de las variables basales entre grupos (mediana, rango intercuartílico)

\begin{tabular}{|c|c|c|c|}
\hline Variables & $\begin{array}{l}\text { Grupo } 1 \\
(n=34)\end{array}$ & $\begin{array}{l}\text { Grupo } 2 \\
(n=38)\end{array}$ & p \\
\hline Edad (años) & $\begin{array}{c}46 \\
(33.75-60.50)\end{array}$ & $\begin{array}{c}56 \\
(49.7-60.5)\end{array}$ & $0.005^{*}$ \\
\hline $\begin{array}{l}\text { Grosor del campo } \\
\text { central }(\mu \mathrm{m})\end{array}$ & $\begin{array}{c}250 \\
(237.50-268)\end{array}$ & $\begin{array}{c}250.50 \\
(232-265.5)\end{array}$ & $0.513^{*}$ \\
\hline $\begin{array}{l}\text { Volumen macular } \\
\left(\mathrm{mm}^{3}\right)\end{array}$ & $\begin{array}{c}10.10 \\
(9.87-10.52)\end{array}$ & $\begin{array}{c}9.90 \\
(9.70-10.30)\end{array}$ & $0.222^{*}$ \\
\hline Sexo femenino (\%) & 52.9 & 63.2 & $0.380^{* *}$ \\
\hline $\begin{array}{l}\text { Hipertensión arterial } \\
(\%)\end{array}$ & 0 & 35.7 & $0.291^{* *}$ \\
\hline
\end{tabular}

regresión lineal para determinar la contribución de la densidad de cada cuadrante a la distribución de la densidad interna; se identificó el percentil del grupo sin diabetes, en el cual se encontraba el percentil 90 del grupo con diabetes, para los cuadrantes con mayor contribución a los cambios de densidad interna. La información se almacenó y analizó en la versión 22 del programa SPSS (Statistical Package for the Social Sciences) para Windows; se consideró significativo un valor de $p<0.05$.

\section{Resultados}

Se evaluaron 72 pacientes con edad de 20 a 77 años $(54.5,43-60), 42$ eran del sexo femenino (58.3\%); 34 no padecían diabetes y 38 sí. El tiempo de evolución de la diabetes fue de 1 a 18 años (5, 2-12); 10 pacientes (13.8\%) padecían hipertensión arterial sistémica. La comparación de las variables basales entre grupos se presenta en la Tabla 1.

La comparación de la densidad vascular entre grupos se muestra en la Tabla 2; en el grupo 2, las densidades interna, completa, superior y nasal fueron menores que en el grupo 1. La comparación de la densidad vascular entre cuadrantes no mostró alguna diferencia estadística en el grupo 1 (Figura 1, $p=0.698$ ) ni en el grupo 2 (Figura 2, $p=0.595$ ).

La Tabla 3 muestra la correlación entre la densidad vascular por cuadrante y la densidad interna por grupo. El análisis de regresión lineal identificó que en el grupo $1(n=34)$, los cuadrantes con mayor contribución a la densidad interna fueron el temporal $(\beta=0.312, p<0.001)$ y el nasal $(\beta=0.286, p<0.001)$. En el grupo $2(\mathrm{n}=38)$, los cuadrantes que más
Tabla 2. Comparación regional de las densidades vasculares entre grupos (mediana, rango intercuartílico)

\begin{tabular}{|c|c|c|c|}
\hline $\begin{array}{l}\text { Densidad } \\
\text { vascular } \\
\left(\mathrm{mm}^{-1}\right)\end{array}$ & $\begin{array}{l}\text { Grupo } 1 \\
(n=34)\end{array}$ & $\begin{array}{l}\text { Grupo } 2 \\
(n=38)\end{array}$ & $p^{*}$ \\
\hline Central & $\begin{array}{c}10.90 \\
(7.70-12.42)\end{array}$ & $\begin{array}{c}8.55 \\
(6.87-11.65)\end{array}$ & 0.066 \\
\hline Interna & $\begin{array}{c}21.85 \\
(20.27-23.02)\end{array}$ & $\begin{array}{c}20.75 \\
(19.47-21.70)\end{array}$ & 0.021 \\
\hline Completa & $\begin{array}{c}20.45 \\
(18.97-21.90)\end{array}$ & $\begin{array}{c}19.55 \\
(18.05-20.42)\end{array}$ & 0.029 \\
\hline Superior & $\begin{array}{c}21.75 \\
(20.40-23.07)\end{array}$ & $\begin{array}{c}21.05 \\
(19.60-21.87)\end{array}$ & $<0.001$ \\
\hline Temporal & $\begin{array}{c}21.95 \\
(20.10-22.90)\end{array}$ & $\begin{array}{c}21.50 \\
(19.80-22.02)\end{array}$ & 0.177 \\
\hline Inferior & $\begin{array}{c}21.40 \\
(20.30-23.02)\end{array}$ & $\begin{array}{c}21.05 \\
(19.50-22.40)\end{array}$ & 0.160 \\
\hline Nasal & $\begin{array}{c}21.50 \\
(20.57-23.52)\end{array}$ & $\begin{array}{c}20.95 \\
(19.75-21.72)\end{array}$ & 0.023 \\
\hline
\end{tabular}

*U de Mann-Whitney

Tabla 3. Correlación entre la densidad vascular por cuadrante y la densidad interna por grupo

\begin{tabular}{|l|c|c|c|c|}
\hline \multirow{2}{*}{ Cuadrante } & \multicolumn{2}{|c|}{ Grupo 1 } & \multicolumn{2}{c|}{ Grupo 2 } \\
\cline { 2 - 6 } & Rho* & $p$ & Rho* & P \\
\hline Superior & 0.249 & $<0.001$ & 0.361 & $<0.001$ \\
\hline Temporal & 0.312 & $<0.001$ & 0.272 & $<0.001$ \\
\hline Inferior & 0.237 & $<0.001$ & 0.285 & $<0.001$ \\
\hline Nasal & 0.286 & $<0.001$ & 0.294 & $<0.001$ \\
\hline
\end{tabular}

*Spearman

contribuyeron a la densidad interna fueron el superior $(\beta=0.361, p<0.001)$ y el nasal $(\beta=0.294, p<0.001)$, los cuales tuvieron una menor densidad vascular que los mismos cuadrantes del grupo 1.

En el análisis de distribución se identificó que los valores del percentil 90 del grupo 2 correspondían a los percentiles 60 y 50 del grupo 1, en los cuadrantes superior y nasal. Además, los valores del percentil 90 del grupo 2 correspondían a los percentiles 60 y 70 del grupo 1, en los cuadrantes inferior y temporal (Tabla 4).

\section{Discusión}

En sujetos sin diabetes, la mayor contribución a la distribución de la densidad vascular parafoveal interna la aportaron los cuadrantes temporal y nasal. No obstante, en los pacientes diabéticos sin retinopatía, los 
Tabla 4. Percentiles de la densidad vascular parafoveal por cuadrantes y grupos

\begin{tabular}{|l|c|c|c|c|c|c|c|c|}
\hline Cuadrante & \multicolumn{2}{|c|}{ Superior } & \multicolumn{3}{c|}{ Nasal } & \multicolumn{2}{c|}{ Inferior } \\
\hline Percentil & Grupo 1 & Grupo 2 & Grupo 1 & Grupo 2 & Grupo 1 & Grupo 2 & Grupo 1 & Grupo 2 \\
\hline 90 & 23.63 & 22.77 & 24.20 & 22.85 & 23.55 & 22.51 & 23.40 & 22.82 \\
\hline 80 & 23.30 & 22.20 & 23.60 & 21.84 & 23.10 & 22.40 & 23.00 & 22.26 \\
\hline 75 & 23.07 & 21.87 & 23.52 & 21.72 & 23.02 & 22.40 & 22.90 & 22.02 \\
\hline 70 & 22.90 & 21.70 & 23.45 & 21.63 & 22.75 & 21.92 & 22.70 & 21.90 \\
\hline 60 & 22.50 & 21.48 & 22.90 & 21.44 & 22.00 & 21.34 & 22.60 & 21.60 \\
\hline 50 & 21.75 & 21.05 & 21.50 & 20.95 & 21.40 & 21.05 & 21.95 & 21.50 \\
\hline 40 & 21.10 & 20.62 & 21.10 & 20.68 & 21.00 & 20.52 & 20.90 & 21.22 \\
\hline 25 & 20.40 & 19.60 & 20.57 & 19.75 & 20.30 & 19.50 & 20.10 & 19.80 \\
\hline
\end{tabular}

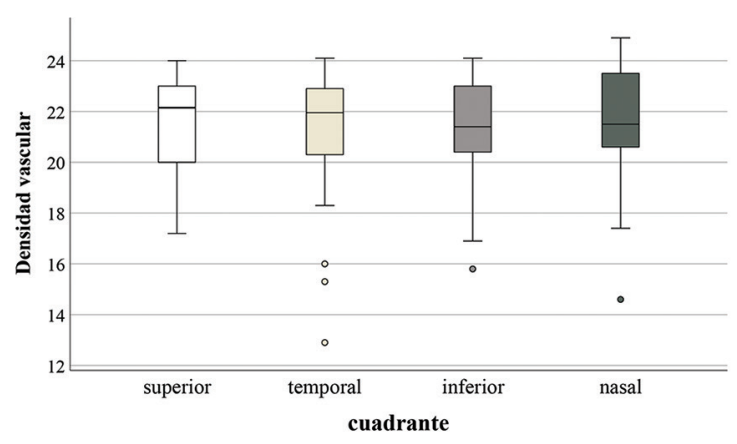

Figura 1. Comparación de la densidad vascular entre cuadrantes en el grupo $1(n=34)$. Densidad vascular en $\mathrm{mm}^{1}$.

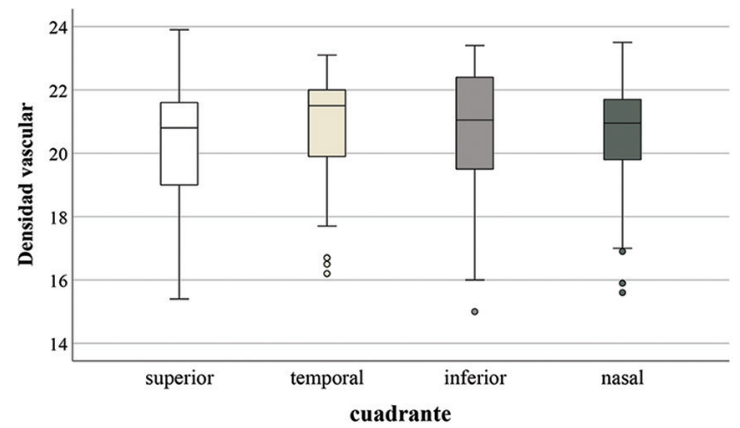

Figura 2. Comparación de la densidad vascular entre cuadrantes en el grupo $2(n=38)$. Densidad vascular en $\mathrm{mm}^{-1}$.

cuadrantes que más contribuyeron fueron el superior y el nasal, cuya densidad vascular fue menor estadísticamente que la del grupo 1.

Un estudio en pacientes de China evaluó la contribución de la densidad vascular por cuadrante para desarrollar retinopatía diabética; para ello se compararon pacientes sin retinopatía con pacientes con retinopatía de distintos grados. Ese estudio empleó un equipo distinto al de la presente investigación, no reportó los valores de densidad vascular por cuadrante ni comparó esos valores con los de sujetos sin diabetes. ${ }^{9}$

Van de Kreeke et al. reportaron los cambios en las capas de la retina y la presencia de retinopatía diabética a través del tiempo; compararon pacientes sin retinopatía y pacientes con retinopatía leve; encontraron cambios en el grosor retiniano, más notorios en el sector temporal. Ese estudio reportó los valores de las capas de la retina por cuadrantes, pero no comparó dichos valores con los de sujetos sin diabetes ni la densidad vascular; ${ }^{10}$ sin embargo, demostró variaciones longitudinales regionales, principalmente en los sitios donde se desarrollaba retinopatía diabética.

Uğurlu también había descrito una reducción de la densidad vascular por cuadrantes entre pacientes diabéticos con y sin retinopatía, pero no comparó sus datos con los valores de sujetos sin diabetes, ni determinó la contribución regional al cambio global. ${ }^{11}$ AttaAllah identificó una reducción de la densidad vascular en los sectores superior e inferior en pacientes diabéticos sin edema, comparados con sujetos sin diabetes; sin embargo, los pacientes con diabetes tenían retinopatía diabética no proliferativa moderada o severa. ${ }^{12}$

La comparación de la densidad vascular entre cuadrantes no mostró diferencias estadísticas en cada grupo, pero la densidad interna disminuyó en el grupo 2. En ese grupo no se presentó una asimetría entre cuadrantes que permitiera identificar al más afectado, porque en todos hubo una reducción que solo fue 
estadística en dos, pero bastó para disminuir la densidad vascular interna.

En nuestro estudio, los cuadrantes que presentaron una reducción estadística de la densidad vascular fueron los que más contribuyeron a los cambios de la densidad interna; la comparación de los percentiles entre grupos mostró que la diferencia no fue mínima, lo cual sugiere que el daño microvascular en dichos cuadrantes no era reciente. Sería conveniente buscar pacientes con daño más temprano para identificar si el efecto es consistente en los cuadrantes que tuvieron reducción de la densidad vascular.

Esperar a que la densidad interna disminuya podría retrasar el diagnóstico de daño microvascular, particularmente cuando se pretende comparar la afección de los capilares retinianos con la de los plexos vasculares de otros órganos..$^{13,14}$

Las fortalezas del estudio fueron la cuantificación automática de los parámetros, la eliminación de la variabilidad interobservador y la señal de intensidad > 7. Una limitación fue que se analizó solo la densidad del plexo superficial no la del plexo profundo, ya que el equipo solo mide automáticamente el primero. Otra limitante fue que si bien en los pacientes del grupo 2 el tiempo de evolución de la diabetes tuvo una mediana de cinco años, 35.7\% presentó hipertensión. Se requerirá ponderar la participación de esta enfermedad para el desarrollo de pérdida capilar retiniana.

En el futuro deberán realizarse estudios longitudinales para detallar el impacto del daño microvascular temprano en la mácula antes de la aparición de la retinopatía diabética. La evaluación regional por cuadrantes podría ser una alternativa para detectar y abordar precozmente la microangiopatía retiniana causada por la diabetes.

\section{Conclusión}

Se encontró una reducción de la densidad vascular interna parafoveal en pacientes diabéticos sin retinopatía, principalmente a expensas de la disminución en los cuadrantes superior y nasal. Este hallazgo sugiere un daño localizado con mayor duración, que deberá confirmarse para detectar una afección microvascular más temprana.

\section{Agradecimientos}

A la empresa Zeiss, México, por el apoyo para la evaluación de los pacientes con el equipo Cirrus 5000 con Angioplex.

\section{Financiamiento}

Los autores no recibieron patrocinio para llevar a cabo este artículo.

\section{Conflicto de intereses}

Los autores declaran que no tuvieron ningún conflicto de interés para el desarrollo ni para la publicación del trabajo.

\section{Responsabilidades éticas}

Protección de personas y animales. Los autores declaran que para esta investigación no se realizaron experimentos en seres humanos ni en animales.

Confidencialidad de los datos. Los autores declaran que siguieron los protocolos de su centro de trabajo sobre la publicación de datos de pacientes.

Derecho a la privacidad y consentimiento informado. Los autores declaran que en este artículo no aparecen datos de pacientes.

\section{Bibliografía}

1. Zheng $Y, H e$ M, Congdon N. The worldwide epidemic of diabetic retinopathy. Indian J Ophthalmol. 2012;60:428-431.

2. Wang W, Lo A. Diabetic retinopathy: pathophysiology and treatments. Int J Mol Sci. 2018;19:1816.

3. Lee J, Rosen R. Optical coherence tomography angiography in diabetes. Curr Diab Rep. 2016;16:123.

4. Gildea D. The diagnostic value of optical coherence tomography angiography in diabetic retinopathy: a systematic review. Int Ophthalmol. 2018;39:2413-2433.

5. Batista M, Borrlli E, Sacconi R, Bandello F, Querques G. Optical coherence tomography angiography in diabetes: a review. Eur J Ophthalmol. 2020;30:411-416.

6. Rosenfeld P, Durbin M, Roisman L, Zheng F, Miller A, Robbins G, et al. ZEISS Angioplex ${ }^{\mathrm{TM}}$ spectral domain optical coherence tomography angiography: technical aspects. Dev Ophthalmol. 2016;56:18-29.

7. Cao D, Yang D, Huang Z, Zeng Y, Wang J, Hu Y, et al. Optical coherence tomography angiography discerns preclinical diabetic retinopathy in eyes with type 2 diabetes without clinical diabetic retinopathy. Acta Diabetol. 2018:55:469-477.

8. Lee MW, Lee WH, Ryu CK, Kim TY, Lim HB, Lee YH, et al. Effects of prolonged type 2 diabetes on the inner retinal layer and macular microvasculature: an optical coherence tomography angiography study. J Clin Med. 2020;9:1849.

9. Xie N, Tan Y, Liu S, Xie Y, Shuai S, Wang W, et al. Macular vessel density in diabetes and diabetic retinopathy with swept-source optical coherence tomography angiography. Graefes Arch Clin Exp Ophthalmol. 2020;258:2671-2679.

10. Van de Kreeke J, Darma S, Chan-Pin-Yin J, Tan H, Abramoff M, Twisk J, et al. The spatial relation of diabetic retinal neurodegeneration with diabetic retinopathy. PLoS One. 2020;15:e0231552.

11. Uğurlu N, Uzel AGT, Sengün A, Yülek F, Özdas D, Tam AA, et al. Evaluation of the correlation between quantitative measurement of the foveal avascular zone and retinal vessel density and outer retinal disruptions in diabetic patients. Turk J Med Sci. 2019;49:1041-1046.

12. AttaAllah HR. Mohamed AAM, Ali MA. Macular vessels density in diabetic retinopathy: quantitative assessment using optical coherence tomography angiography. Int Ophthalmol. 2019;39:1845-1859.

13. Farrah TE, Dhillon B, Keane PA, Webb DJ, Dhaun N. The eye, the kidney, and cardiovascular disease: old concepts, better tools, and new horizons. Kidney Int. 2020;98:323-342.

14. Wang W, He M, Gong X, Wang L, Meng J, Li Y, et al. Association of renal function with retinal vessel density in patients with type 2 diabetes by using swept-source optical coherence tomography angiography. $\mathrm{Br} \mathrm{J}$ Ophthalmol. 2020;104:1768-1773. 